\title{
Deficiency of VCP-Interacting Membrane Selenoprotein (VIMP) Leads to G1 Cell Cycle Arrest and Cell Death in MIN6 Insulinoma Cells
}

\author{
Lili Men ${ }^{\mathrm{a}} \quad$ Juan Sun ${ }^{\mathrm{b}} \quad$ Decheng Ren ${ }^{\mathrm{b}}$ \\ aDepartment of Endocrinology, First Affiliated Hospital of Dalian Medical University, Dalian, China, \\ ${ }^{b}$ Department of Medicine, The University of Chicago, Chicago, USA
}

\section{Key Words}

$\beta$-cell death $\bullet$ UPR $•$ Cell cycle $\bullet$ Insulin secretion $•$ MIN6 cells

\begin{abstract}
Background/Aims: VCP-interacting membrane selenoprotein (VIMP), an ER resident selenoprotein, is highly expressed in $\beta$-cells, however, the role of VIMP in $\beta$-cells has not been characterized. In this study, we studied the relationship between VIMP deficiency and $\beta$-cell survival in MIN6 insulinoma cells. Methods: To determine the role of VIMP in $\beta$-cells, lentiviral VIMP shRNAs were used to knock down (KD) expression of VIMP in MIN6 cells. Cell death was quantified by propidium iodide (PI) staining followed by flow cytometric analyses using a FACS Caliber and FlowJo software. Cell apoptosis and proliferation were determined by TUNEL assay and Ki67 staining, respectively. Cell cycle was analyzed after PI staining. Results: The results show that 1 ) VIMP suppression induces $\beta$-cell apoptosis, which is associated with a decrease in $\mathrm{Bcl}-\mathrm{xL}$, and the $\beta$-cell apoptosis induced by VIMP suppression can be inhibited by overexpression of $\mathrm{Bcl}-\mathrm{xL} ; 2$ ) VIMP knockdown (KD) decreases cell proliferation and $\mathrm{G} 1$ cell cycle arrest by accumulating p27 and decreasing E2F1;3) VIMP KD suppresses unfolded protein response (UPR) activation by regulating the IRE1 $\alpha$ and PERK pathways; 4) VIMP KD increases insulin secretion. Conclusion: These results suggest that VIMP may function as a novel regulator to modulate $\beta$-cell survival, proliferation, cell cycle, UPR and insulin secretion in MIN6 cells.
\end{abstract}

\section{Introduction}

The central defect in both type 1 and type 2 Diabetes mellitus is insufficient insulin supply resulting from $\beta$-cell dysfunction and/or reductions in $\beta$-cell mass [1]. $\beta$-cell mass is highly dynamic and tightly regulated by $\beta$-cell proliferation, apoptosis, and neogenesis to meet the body's demand for insulin [2]. Excessive $\beta$-cell apoptosis and reduced $\beta$-cell 


\section{Cellular Physiology Cell Physiol Biochem 2018;51:2185-2197 \begin{tabular}{ll|l} 
and Biochemistry & $\begin{array}{l}\text { DOl: 10.1159/000495865 } \\
\text { Publisned onlne: } 7 \text { December } 2018\end{array}$ & $\begin{array}{l}\text { O } 2018 \text { The Author(s). Published by S. Karger AG, Basel } \\
\text { www.karger.com/cpb }\end{array}$ \\
\cline { 2 - 3 }
\end{tabular}

proliferation are important contributors to diabetes development [3]. Therefore, inhibiting $\beta$-cell loss and increasing $\beta$-cell number could prevent diabetes progression. Currently, available antidiabetic medicines are mainly targeting insulin secretion and action or glucose absorption [4], and newer interventions need to be found to promote $\beta$-cell survival, proliferation, and neogenesis.

Mounting evidence indicates that $\beta$-cell dysfunction and apoptosis can cause endoplasmic reticulum (ER) stress and inappropriate activation of the unfolded protein response (UPR). When too many misfolded proteins accumulate in the ER, ER homeostasis is disrupted and ER stress ensues. ER stress activates the cytoprotective UPR, which acts to relieve ER stress and restore ER homeostasis [5]. The UPR has three pathways: 1) protein kinase RNA-like ER kinase (PERK)-eukaryotic translation initiation factor $2 \alpha$ (eIF2 $\alpha$ ) and ATF4 pathway; 2) inositol-requiring enzyme 1 alpha (IRE1 $\alpha$ )-X-box binding protein 1 (XBP1) pathway, and 3) transcription factor 6 alpha (ATF6 $\alpha$ ) pathway. Through these signaling cascades, the UPR attempts to maintain ER homeostasis and limit ER stress [6]. Studies suggest that the loss of adaptive UPR has been associated with altered $\beta$-cell function and increased cell death leading to progressive diabetes [7]. For example, $\beta$-cell specific Xbp1 mutant mice exhibited hyperglycemia, islet cell loss and inhibited $\beta$-cell proliferation [8]. PERK-deficient mice cause permanent neonatal diabetes due to the severe defects in fetal/neonatal $\beta$-cell proliferation, an increase in $\beta$-cell death and impaired insulin secretion $[9,10]$.

Valosin-containing protein (VCP)-interacting membrane protein (VIMP, selenoprotein $\mathrm{S}$, Tanis) is an ER resident selenoprotein. VIMP is identified to form a complex with SelK, p97(VCP), Derlin-1 or -2, and E3 ligases, which contribute to the formation of ERassociated protein degradation (ERAD) for maintaining protein quality control [11]. VIMP protects macrophages against ER stress-induced cellular dysfunction [12] and is involved in inflammation and management of oxidative stress $[13,14]$. Genetic studies of VIMP polymorphisms have further revealed associations with metabolic risk factors [15]. VIMP also plays a role in regulating glucose homeostasis in an animal model of type 2 diabetes mellitus and in human diabetic subjects $[16,17]$. However, the role of VIMP in $\beta$-cells is not identified. The present study was undertaken to define the role of VIMP in $\beta$-cell survival and proliferation, and to identify the mechanism of $\beta$-cell apoptosis induced by VIMP suppression in MIN6 cells.

\section{Materials and Methods}

MIN6 cells culture, Quantification of mRNA levels, and lentivirus-mediated short hairpin RNA (shRNA) expression

MIN6 cell culture, RNA isolation, first-strand cDNA synthesis, and preparation of pLKO.1-VIMP shRNA lentivirus were all performed as previously described [18]. TaqMan assay numbers (Invitrogen) were as follows: Bcl-xL (Mm00437783_m1); Bcl-2(Mm00477631_m1); p27 (Mm00438168_m1), VIMP (Mm01318786_m1). Lentiviral vector (TRCN0000086031) was purchased from Thermo Scientific. VIMP shRNAs (RMM4534) and a scrambled shRNA for non-target control were designed and synthesized by GE Dharmacon. The production of recombinant shRNAs lentivirus was performed by co-transfection into the HEK293T packing cell line, as described previously [19]. Lentivirus was added to the medium on day 0. Pan-caspase inhibitor benzyloxycarbonyl-Val-Ala- Asp-fluoromethylketone (Z-VAD) $(20 \mu \mathrm{mol} / \mathrm{L})$ was added to the medium $2 \mathrm{~h}$ prior to treating MIN6 cells by VIMP shRNA lentivirus. Z-VAD was added to the cells on day 0 and 3.

\section{Western Blot}

Cells were lysed with RIPA Buffer (Pierce) consisting of $25 \mathrm{mM}$ Tris- $\mathrm{HCl}$ (pH 7.6), $150 \mathrm{mM} \mathrm{NaCl}$, $1 \%$ NP-40, $1 \%$ sodium deoxycholate and $0.1 \%$ SDS. Additionally, protease inhibitor and phosphatase inhibitor cocktail were supplemented to the lysis buffer. The blots were probed with primary antibodies against Cleaved casp3 (9661), Puma (7467), Bcl-xL (2762), CDK6 (3136S), Cyclin D1 (2922), p-S6 (5364), S6 (2217), p-4E-BP (9451), 4E-BP (9644), p-eIF2a (9721), eIF2a (9722), p-Rb and Rb (9969), p-AKT and 


\section{Cellular Physiology Cell Physiol Biochem 2018;51:2185-2197 and Biochemistry \begin{tabular}{l|l} 
DOI: 10.1159/000495865 & $\begin{array}{l}\text { O } 2018 \text { The Author(s). Published by S. Karger AG, Basel } \\
\text { wwww.karger.com/cpb }\end{array}$
\end{tabular} \\ Men et al.: VIMP Deficiency Causes $\beta$-Cell Death}

AKT (9916), IRE1 $\alpha$ (3294), CHOP(2895) were purchased from the Cell Signaling Technology Inc; VIMP (15591-1-AP), CDK4 (11026-1-AP), Cyclin E1 (11554-1-AP), CDK2 (10122-1-AP), Cyclin A2 (18202-1-AP) primary antibody were from Proteintech; Bax (sc-493), Bad (sc-943), Mcl-1 (sc-819), Cyclin B1 (sc-752), P21 (sc-471), P27 (sc-1641), E2F1 (sc-193), sXBP1 (sc-7160), and Grp78 (sc-1050) were from Santa Cruz Biotechnology; Bim (202000) were from Calbiochem; Bcl-2 (554218) were from Pharmingen; Bak (06536) were from EMD Millipore; ATF4 (ARP37017_P050) were from Aviva systems biology; Actin (A1978) were from Sigma-Aldrich. All the antibodies used in the experiments were diluted by 1:1000. Antibody detection was accomplished using an enhanced chemiluminescence method and LAS-3000 imaging system (FUJIFILM).

\section{Quantitation of cell death}

Cell death was quantified by propidium iodide (PI) staining. 4 or 5 days after infection with VIMP shRNA lentivirus, MIN6 cells were harvested and then assessed by PI staining [20], followed by flow cytometric analyses using a FACS Caliber (BD Bioscience) and FlowJo software.

\section{TUNEL labeling}

TUNEL assay was used to measure cell apoptosis in VIMP KD MIN6 cells. In brief, cells were seeded on glass coverslips in 4-well dishes, infected with recombinant shRNA lentivirus on day 0 . Then the apoptosis of cells at day 3 was analyzed via TUNEL assay using Cell Death Detection Kit (Roche Diagnostics) following the manufacturer's recommended procedure. The samples were stained with DAPI to visualize total cells. TUNEL-positive cells were counted and normalized to total MIN6 cells.

\section{Ki67 staining}

Cell proliferation was assessed by Ki67 staining. MIN6 cells were seeded on glass coverslips in 4-well dishes and infected with VIMP shRNA or scrambled shRNA recombinant lentivirus. After infection for $48 \mathrm{~h}$, cell proliferation was determined by Ki67 staining. Briefly, cells were fixed with $10 \%$ formalin for 25 min and then permeabilized in PBS containing $0.2 \%$ Triton X-100 for $5 \mathrm{~min}$ at room temperature. The cells were blocked using 5\% protease-free BSA in PBS for 30min at room temperature followed by incubation with anti-Ki67 primary antibody (652401; Biolegend) at $4^{\circ} \mathrm{C}$ overnight and then with Alexa Fluor 488-labeled secondary antibody (Thermo Scientific) for $1 \mathrm{~h}$, and subsequently stained with DAPI for $5 \mathrm{~min}$ at room temperature before detection by fluorescence microscopy.

\section{Retrovirus infection}

Human Bcl-xL was cloned into the retroviral expression vector MSCV-IRES-GFP (pMIG) (Addgene). The production of amphotropic retroviruses using the 293GPG packing cell line was performed as described previously [21]. Retroviruses were added to the medium on day 0. MIN6 cells were infected with these retroviral vectors at multiplicity of infection of 10 .

\section{Analysis of cell cycle distribution}

3 days after VIMP KD, MIN6 cells were trypsinized and harvested by centrifugation, and then fixed in $75 \%$ ethanol at $-20^{\circ} \mathrm{C}$ overnight. Fixed cells were washed with PBS, resuspended with $20 \mu \mathrm{g} / \mathrm{ml}$ RNase A in PBS and incubated for $10 \mathrm{~min}$ at room temperature. Subsequently, cells were stained with $25 \mu \mathrm{g} / \mathrm{ml}$ PI for $30 \mathrm{~min}$ at room temperature in the dark and subjected to analysis via a BD FACS Caliber.

\section{Insulin secretion}

4 days after culture with control or VIMP shRNA lentivirus, MIN6 cells were washed twice in KRHB buffer and were pre-incubated with $2.8 \mathrm{mM}$ glucose for $30 \mathrm{~min}$ prior to switching to KRHB culture medium containing $2.8 \mathrm{mM}$ glucose and $16.7 \mathrm{mM}$ glucose for 60 minutes at $37^{\circ} \mathrm{C}$. Insulin concentration in the medium was measured by ELISA assay. 


\section{Cellular Physiology Cell Physiol Biochem 2018;51:2185-2197 \begin{tabular}{ll|l} 
and Biochemistry Published onIIne: 7 December 2018 & $\begin{array}{l}\text { (c) } 2018 \text { The Author(s). Published by S. Karger AG, Basel } \\
\text { www.karger.com/cpb }\end{array}$ \\
\hline
\end{tabular}

Statistical analysis

The two-tailed unpaired student $t$ test was used to assess the statistical significance of the difference between two sets of data. ${ }^{*} P<0.05$, ${ }^{* *} P<0.01$, and ${ }^{* * *} P<0.001$ were considered significant compared with the control group. Variables were presented as mean \pm standard deviation. Experiments were repeated three times

\section{Results}

Reduced VIMP expression leads to apoptosis in MIN6 insulinoma cells

To define the role of VIMP in $\beta$-cells, we used three different lentiviral VIMP shRNAs to knock down expression of VIMP in MIN6 cells. The results showed that all three shRNAs significantly decreased VIMP protein levels. The protein levels of VIMP were decreased by $95 \%$ and $92 \%$ (both $P<0.001$ ) in MIN6 cells after infection with lentiviral VIMP shRNA 1\# and $3 \#$ for 2 days, respectively (Fig. 1A). The impact of VIMP KD on the death of MIN6 cells was next studied and it was determined that VIMP KD significantly increased cell death in MIN6 cells by day 4 (Fig. 1B). The effect of VIMP KD on $\beta$-cell viability was further explored to quantify $\beta$-cell death by using PI staining followed by flow cytometry. After infection with VIMP shRNA lentivirus, the proportion of PI staining-positive cells was increased from $55.2 \%$ on day 4 to $77.1 \%$ on day $5(P<0.001)$ (Fig. $1 C)$. To further define the effects of VIMP suppression on $\beta$-cell survival, a terminal dUTP nick end labeling (TUNEL) assay was used to measure cell apoptosis. The results showed that on day 3 the number of TUNEL positive cells was $24.3 \%$ in VIMP KD, which is much more than the number of $5.3 \%$ in control cells $(P<0.001)$ (Fig. 1D), indicating that VIMP suppression significantly increased cell death. These changes induced by VIMP suppression were associated with an increase in cleavedcaspase3 (C-casp3) (Fig. 1E). Z-VAD, a pan-caspase inhibitor, significantly inhibited the increase of C-casp3 protein levels in Z-VAD/VIMP KD cells (Fig. 1F). VIMP KD did not affect cleavage of caspase 9 and caspase 12 (Fig. 1G). These results indicate that reduced VIMP expression leads to apoptotic $\beta$-cell death.

\section{Bcl-xL mediates $\beta$-cell apoptosis induced by VIMP suppression}

Since Bcl-2 family members are widely involved in apoptotic cell death, we determined whether VIMP KD induced the changes in the protein levels of Bcl-2 family members, including anti-apoptotic members and pro-apoptotic members. For anti-apoptotic members, we measured the protein levels of Bcl-2, Bcl-xL and Mcl-1; for pro-apoptotic members we measured the protein levels of Bim, Puma, Bax and Bak. The results showed that VIMP KD resulted in a significant decrease in Bcl-xL protein levels in MIN6 cells (Fig. 2A). Surprisingly, VIMP KD induced a decrease rather than an increase in Bim protein levels (Fig. 2A). Protein levels of Bcl-2, Mcl-1, Puma, Bax and Bak were not altered by VIMP KD (Fig. 2A). Consistently, mRNA levels of Bcl-xL were markedly decreased, while Bcl-2 expression was unchanged in VIMP KD cells (Fig. 2B). Bcl-xL overexpression (OE) significantly inhibited the increase in C-casp3 protein levels induced by VIMP KD in Bcl-xL OE /VIMP KD cells (Fig. 2C). Bcl-xL $\mathrm{OE}$ also significantly inhibited the increase in the number of PI staining positive cells. The cells staining positive for PI were decreased from $56.1 \%$ in VIMP KD group to $25.8 \%$ in Bcl-xL OE /VIMP KD group $(P<0.001)$ (Fig. 2D). Collectively, these findings show that VIMP suppression decreases Bcl-xL and this is associated with an increase in $\beta$-cell apoptosis in MIN6 cells.

VIMP suppression decreases $\beta$-cell proliferation and causes $G 1$ arrest

To determine whether VIMP KD affects the proliferation, we measured proliferative marker Ki67 staining. The results showed that proliferation of MIN6 cells was decreased by $25 \%$ after VIMP KD compared with control cells (Fig. 3A). To elucidate the mechanism by which VIMP modulates cell proliferation, we analyzed the phosphorylation of AKT. The results showed that phosphorylation levels of Akt at Ser-473 (p-AKT473) were decreased 
Fig. 1. VIMP KD induces cell death in MIN6 cells. A: Effect of VIMP knockdown (KD) was assessed by western blot. 2 days after infection with lentivirus c o n t a i n i n g scrambled shRNA as control or 3 different lentiviral VIMP shRNAs, immunoblotting analysis was used to verify VIMP KD efficiency. B: MIN6 cells were observed under inverted phase contrast microscope after infection with VIMP lentiviral shRNA for 4 days. Arrows show the dead cells. Scale bar $20 \mu \mathrm{m}$. C: C-Casp3 and VIMP protein levels in VIMP KD cells. 4 days after VIMP $\mathrm{KD}$, the levels of C-Casp3 and VIMP in MIN6 cells were determined by Western blots. D: Cell death was determined by PI staining. 4 and 5 days after MIN6
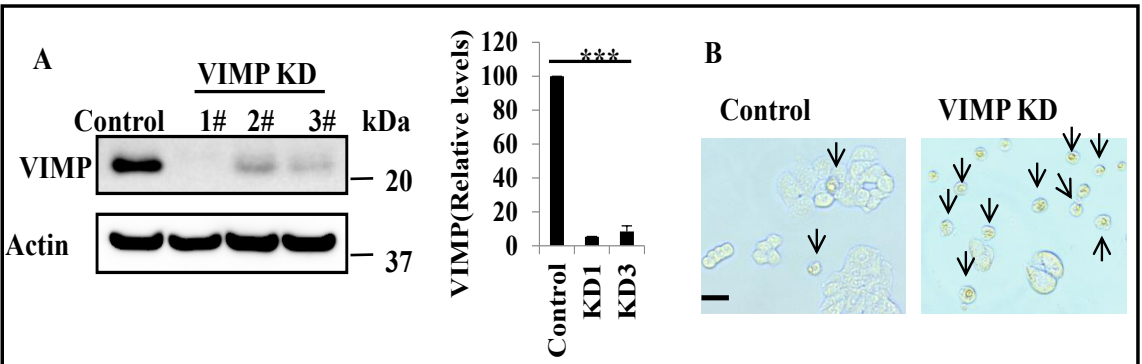

C
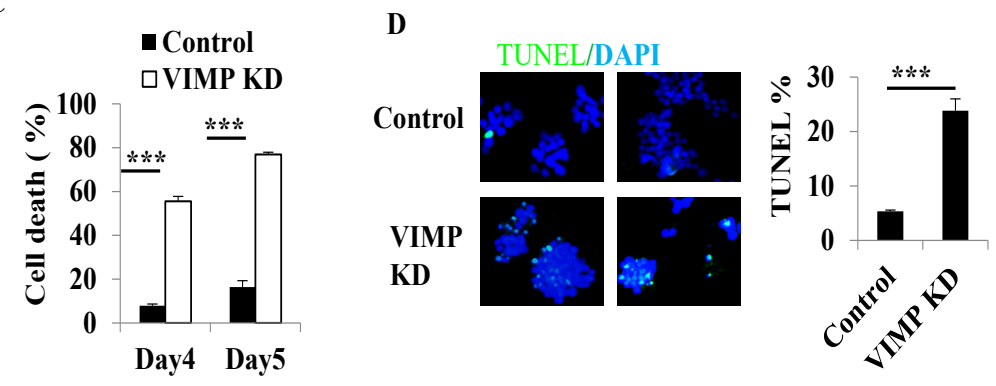

E

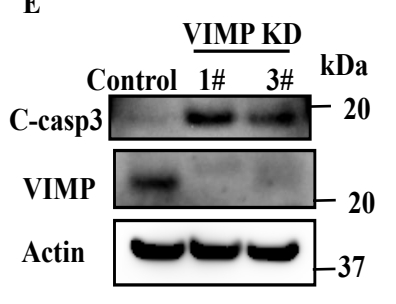

F

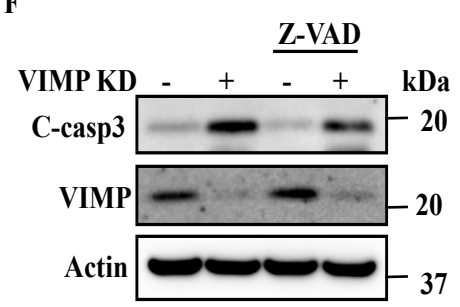

G VIMP KD

\section{Control 1\# 3\#}

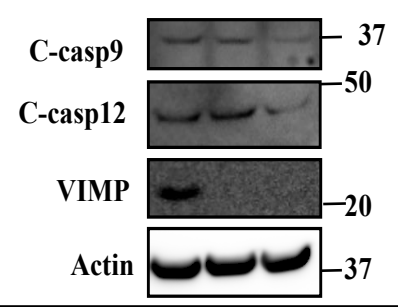

cells were infected with VIMP shRNA, percentage of cell death was analyzed via FACS. E: TUNEL labeling of VIMP KD MIN6 cells. 3 days after infection with VIMP shRNA lentivirus, apoptotic cells were assayed by TUNEL staining. Quantitative TUNEL data are shown. Scale bar $20 \mu \mathrm{m}$. F: The effect of caspase inhibitor (Z-VAD) on C-casp3 and VIMP protein levels in control and VIMP KD MIN6 cells. $20 \mu \mathrm{M}$ Z-VAD was added to the medium on day 0 and 3 prior to infection with VIMP shRNA lentivirus. The cells were harvested on day 4. Values in panel (A), (C) and (D) are means \pm SEM $(n=3) . * * * P<0.001$ versus control. G: Effect of VIMP KD on the other caspases. 4 days after VIMP KD, the levels of cleaved caspase9 and caspase 12 in MIN6 cells were determined by Western blots. 
Fig. 2. Bcl-xL is $\mathrm{A}$ decreased after VIMP suppression in MIN6 cells. A: Western blot of VIMP KD cells. 4 days after VIMP KD in MIN6 cells, immunoblot analysis was performed to determine the protein levels of VIMP, Bim, Puma, Bcl-xL, Bcl-2, Mcl-1, Bak, and Bax. B: Bcl-xL and Bcl-2 mRNA levels in VIMP KD cells. 4 days after VIMP KD in MIN6 cells, Bcl-xL and Bcl2 mRNA levels were measured by QRTPCR. C: Western blot of VIMP KD/Bcl-xLoverexpressing (OE) cells. 4 days after VIMP KD and/or Bcl$\mathrm{xL}$ OE in MIN6cells, immunoblot

C-cas3, Bcl-xL, and VIMP proteins were determined. Values are means \pm SEM $(\mathrm{n}=$

3), ${ }^{* *} \mathrm{P}<0.01,{ }^{* *} \mathrm{P}<0.001$ versus control. D: Cell death was determined by PI staining in VIMP KD/Bcl-xL OE cells. The percentage of cell death was shown. Values are means \pm SEM $(n=3),{ }^{* * *} \mathrm{P}<0.001$ versus control, \#\#\#P<0.001 versus VIMP KD group.

while p-AKT308 was unchanged in VIMP KD cells (Fig. 3B). The results indicate that AKT signaling pathways are involved in mediating the effects of VIMP on $\beta$-cell proliferation.

To further identify the mechanism responsible for reduced proliferation of VIMP KD cells, we studied the effect of VIMP on cell mitosis and cell cycle-related proteins. VIMP KD cells showed an increase in the number of cells in $G_{1}$ phase and a concomitant decrease in G2-M transition, confirming the G1-phase arrest (Fig. 3C). As shown in Fig. 4D, cyclin D1 protein levels in $G_{1}$ phase, cyclin $E 1$ in the late $G_{1}$ phase and cyclin $A 2$ in the $S$-phase transition were decreased in VIMP KD cells. VIMP KD had no significant effect on protein levels of cyclin B1. There was no significant difference in cyclin-dependent protein kinases CDK6 and CDK2 between control and VIMP KD cells (Fig. 3D). Surprisingly, CDK4 was slightly increased in VIMP KD cells. To further identify the mechanism of cell cycle arrest, the protein levels of cell cycle inhibitors such as retinoblastoma protein (Rb), p21 and p27 were determined. Indeed, the phosphorylation of $\mathrm{Rb}$ at two sites was strongly increased in the late $\mathrm{G}_{1}$ phase in control cells, whereas VIMP KD inhibited the phosphrylation of Rb (Fig. 3E). As expected, E2F1, which is repressed by active phosphorylated $\mathrm{Rb}$, was increased in control cells but decreased in VIMP KD cells (Fig. 3E). p27 protein levels were markedly increased in VIMP KD cells, but p21 protein levels were not affected by VIMP suppression (Fig. 3F). The S-phase kinase-associated protein 2 (Skp2) is the major p27-ubiquitin ligase. The S-phase kinaseassociated protein 1 (Skp1) can become complexed with Skp2 to regulate 27 degradation. To 


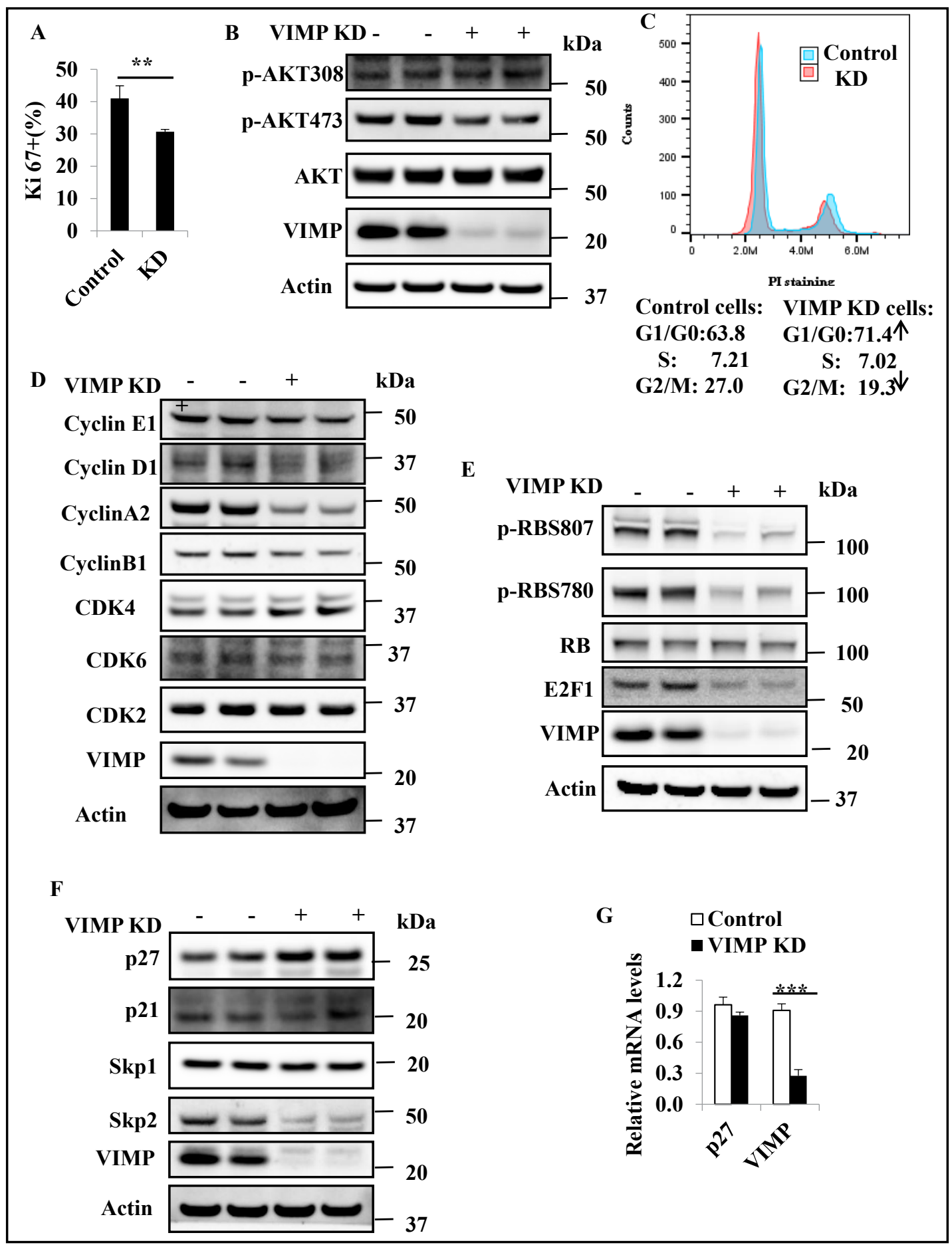

Fig. 3. VIMP KD decreases $\beta$-cell proliferation and causes G1 arrest in MIN6 cells. A: Ki-67 staining of $\beta$-cell. After $48 \mathrm{~h}$ infection with VIMP lentiviral shRNA, Ki-67 staining for proliferation cell nuclei and subsequent DAPI staining for all the nuclei were performed in MIN6 cells. Quantitative Ki-67 staining data were shown. Values are means \pm SEM, ${ }^{* *} \mathrm{P}<0.01$ versus control cells. B: Effects of VIMP KD on p-AKT, p-S6, and p-4E-BP. 4 days after infection with VIMP shRNA lentivirus, proteins levels of p-AKT308, p-AKT473, AKT, p-S6, S6, p-4E-BP and 4E-BP were determined by Western blot. ( C - G) Cell cycle-related proteins levels in control and VIMP KD cells. 4 days after VIMP KD, the protein levels of cyclins and CDKs $(\mathrm{C}+\mathrm{D})$, cell cycle and $\mathrm{CDK}$ inhibitors $(\mathrm{E}+\mathrm{F})$ and p27 mRNA levels in control and VIMP KD cells were determined by Western blot and qRT-PCR. 
determine whether $\mathrm{p} 27$ is regulated at a post-translational or transcriptional level, we measured the protein levels of Skp1 and Skp2. The results showed that Skp2 were markedly decreased in VIMP KD cells and Skp1were not affected by VIMP KD (Fig. 3F). The mRNA levels of $\mathrm{p} 27$ were unchanged in VIMP KD cells (Fig. 3G). These results indicate that VIMP KD causes p27 protein accumulation by increasing p27 stabilization. According to these findings, we can conclude that VIMP $\mathrm{KD}$ in MIN6 cells results in remarkable decrease in proliferation and induces cell cycle G1 arrest in MIN6 cells.

\section{VIMP modulates UPR}

Given that activation of ER stress is involved in cell death and cell proliferation, and that VIMP has been shown to modulate ER stress, we determined whether VIMP-regulated cell death and cell proliferation are associated with altered ER stress. First, we examined the effect of ER stress agents, tunicamycin (TC), an inhibitor of N-glycosylation, and thapsigargin (TG), an inhibitor of ER $\mathrm{Ca}^{2+}$ ATPase activity, on VIMP expression. Induction of ER stress by TC- or TG-induced ER stress was indicated by an increase in the ER stress-response proteins IRE1 $\alpha$, spliced XBP1 (sXBP1), PERK, p-eIF2a, and CHOP (Fig. 4A and B). TC or TG treatment induced an increase in VIMP protein levels with maximum induction at $10 \mu \mathrm{g} / \mathrm{ml} \mathrm{TC}$ or $1.0 \mu \mathrm{M}$ TG (Fig. 4A). $5 \mu \mathrm{g} / \mathrm{ml} \mathrm{TC}$ or $0.5 \mu \mathrm{M}$ TG induced maximal VIMP protein levels after $8 \mathrm{~h}$ of treatment. Cleaved ATF6 proteins cannot be detected by commercial antibodies in MIN6 cells. These results clearly show that in MIN6 cells ER stress can regulate VIMP expression and VIMP expression dynamically responds to ER stress.

To further determine whether VIMP KD affects UPR activation, we measured the protein levels of UPR markers in VIMP KD MIN6 cells. As shown in Fig. 5A, VIMP KD induced a decrease in the protein levels of IRE1 $\alpha$, sXBP-1, PERK, p-eIF2a, eIF2a and ATF4. Grp78 protein levels were not altered by VIMP KD. We further determined the role of VIMP in TC- or TG-induced ER stress in MIN6 cells. VIMP KD leads to a significant decrease in protein levels of sXBP1, PERK, p-eIF2a, eIF2a, ATF4 and CHOP in TC- or TG-treated MIN6 cells (Fig. 5B). These results suggest that reduced VIMP expression induces UPR activation defect. 
Fig. 5. VIMP KD decreases UPR activation. A: The protein levels of ER stress markers in control and VIMP KD cells. 4 days after VIMP KD in MIN6 cells, the protein levels of IRE1 $\alpha$, sXBP1, PERK, p-eIF2a, eIF2a, ATF4, Grp78 were determined by Western blot. B: The effects of TC or TG on ER stress markers in

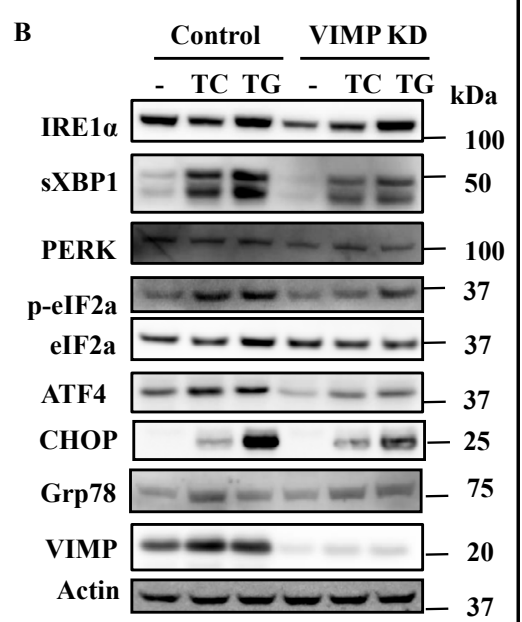
VIMP KD MIN6 cells.

After treatment with $10 \mu \mathrm{g} / \mathrm{ml}$ TC or $1.0 \mu \mathrm{M}$ TG for $8 \mathrm{~h}$ in VIMP KD MIN6 cells, the protein levels of IRE1 $\alpha$, sXBP1, PERK, p-eIF2a, eIF2a, CHOP, Grp78, and VIMP were determined by Western blot. C: The model that VIMP deficiency leads to UPR inactivation, an increase in $\beta$-cell death and a decrease in $\beta$-cell proliferation.

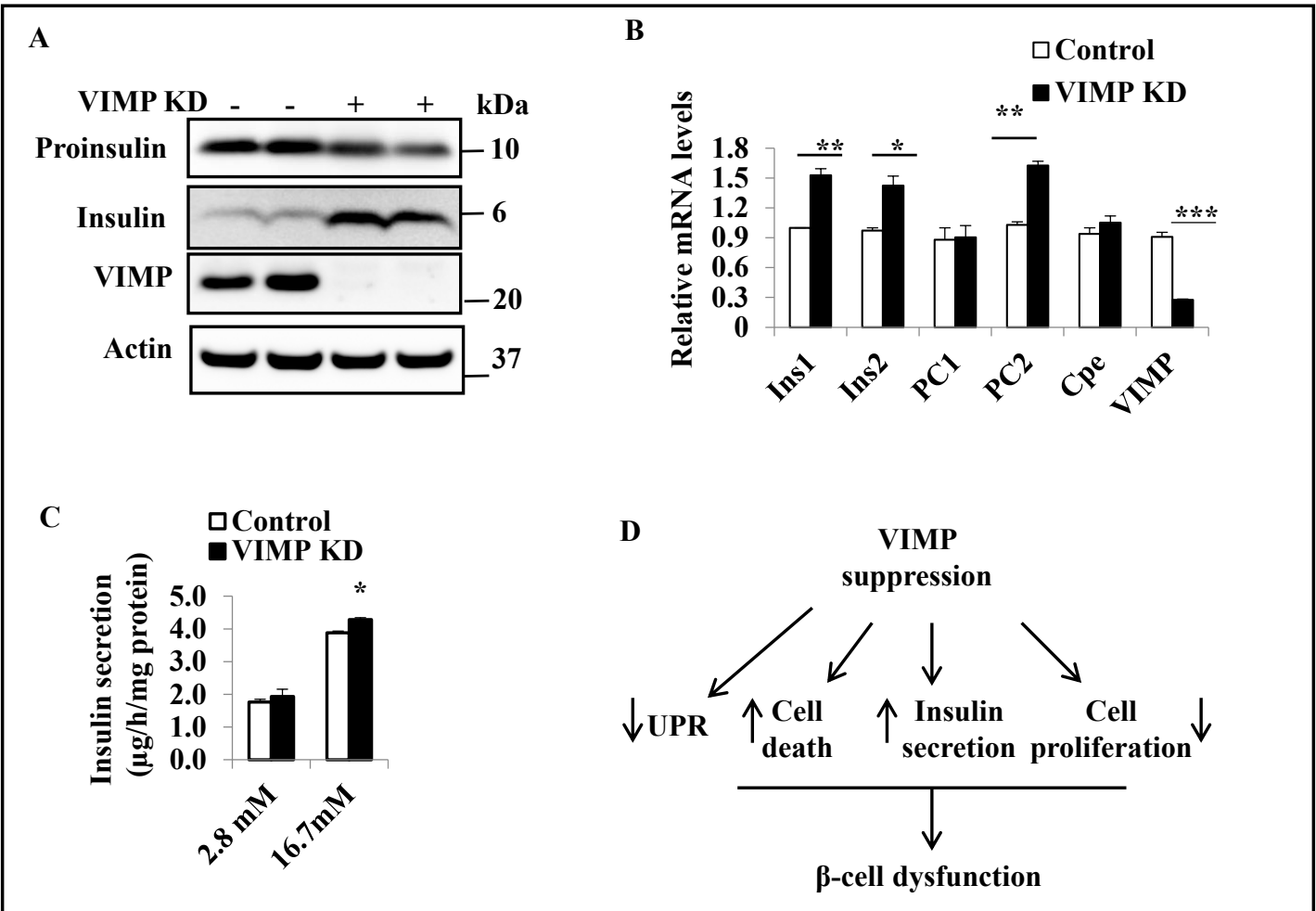

Fig. 6. VIMP KD increases insulin secretion. A. VIMP KD induces an increase in insulin production. 4 days after VIMP KD in MIN6 cells, the protein levels of proinsulin and insulin were determined by Western blot. B. mRNA levels of insulin were increased in VIMP KD MIN6 cells. 4 days after VIMP KD in MIN6 cells, mRNA levels of the genes were determined by qRT-PCR. Values are means \pm SEM $(n=3)$. ${ }^{*} P<0.05$ versus control. C. VIMP KD induces an increase in insulin production. 2 days after VIMP KD in MIN6 cells, the insulin secretion from MIN6 cells in $2.8 \mathrm{mM}$ and $16.7 \mathrm{mM}$ glucose were determined by ELISA. D: The model that VIMP deficiency leads to UPR inactivation, an increase in $\beta$-cell death and insulin secretion, and a decrease in $\beta$-cell proliferation, leading to $\beta$-cell dysfunction in MIN6 cells. 


\section{Cellular Physiology $\quad$ Cell Physiol Biochem 2018;51:2185-2197 \begin{tabular}{l|l|l} 
and Biochemistry Published onIIne: 7 December 2018 & $\begin{array}{l}\text { (c) } 2018 \text { The Author(s). Published by S. Karger AG, Basel } \\
\text { www.karger.com/cpb }\end{array}$ \\
\hline
\end{tabular}

\section{VIMP regulates insulin secretion}

To determine whether VIMP also regulates insulin secretion, we determined the protein levels of insulin in VIMP KD MIN6 cells. The results showed that VIMP induced a decrease in proinsulin and an increase in insulin (Fig. 6A). The mRNA levels of Ins1 and Ins2 were both increased after VIMP KD (Fig. 6B). Proprotein convertase 2 (PC2) was also increased. Carboxypeptidase E (CPE) related to insulin biosynthesis was not altered (Fig. 6B). In addition, insulin secretion was increased in VIMP KD MIN6 cells after incubation with $16.7 \mathrm{mM}$ glucose for $1 \mathrm{~h}$ (Fig. 6C). Fig. 6D presents a model in which VIMP modulates UPR activation, $\beta$-cell survival and proliferation, and insulin secretion. VIMP KD results in a decrease in UPR activation and $\beta$-cell proliferation, and an increase in $\beta$-cell death and insulin secretion in MIN6 cells.

\section{Discussion}

In this study, we found that VIMP deficiency induced $\beta$-cell apoptotic cell death associated with a decrease in Bcl-xL in MIN6 cells. Overexpression of the Bcl-xL significantly inhibited VIMP KD-induced $\beta$-cell apoptosis. These results suggest that VIMP KD leads to an imbalance of the Bcl-2 family members in favor of the pro-apoptotic pathway. In addition, Perk $^{-/-}$or eIF2a ${ }^{-/-}$cells were sensitive to ER stress-induced apoptosis [22-25]. Therefore, the decrease in PERK and p-eIF2a induced by VIMP KD could also contribute to $\beta$-cell apoptosis in MIN6 cells.

Besides cell death, cell proliferation in MIN6 was also regulated by VIMP. The replication of $\beta$-cells is controlled by a network of cell-cycle regulators [26-28]. VIMP deficiency in MIN6 cells induces G1 cell cycle arrest, which is related to imbalance of cell-cycle regulators. For example, VIMP KD decreased protein levels of cyclin D1, cyclin E1 and cyclin A2. VIMP KD also induced a decrease in phosphorylation of $\mathrm{Rb}$ at two sites, which resulted in the inhibition of E2F1 activity. p27 inhibits the cyclin/CDK complex and also blocks the G1/S-phase transition $[29,30]$. Skp2 is the major p27-ubiquitin ligase. VIMP KD induces an increase in p27 due to the reduction in Skp2 in MIN6 cells. We also observed a significant decrease in p-AKT473. In addition to the role in survival, Akt is also involved in cell cycle regulation by preventing GSK$3 \beta$-mediated phosphorylation and degradation of cyclin D1 [31] and by negatively regulating p27 [32]. Decreased Akt phosphorylation at Ser473 might cause an increase in p27 protein levels [33]. Therefore, the reduction in cyclin D1 and accumulation of p27 induced by VIMP KD may also be related to the decrease in AKT activation.

Since VIMP protein is present in the ER, we assume that VIMP regulates $\beta$-cell survival and proliferation likely by modulating ER stress and UPR. During proinsulin synthesis, the $\beta$-cell PERK-eIF2a pathway is a key pathway for maintaining the ER homeostasis [25]. The loss of PERK expression in humans and mice induces diabetes through apoptotic $\beta$-cell death arising from failure to properly regulate the UPR $[9,34]$. IRE1 $\alpha$-sXBP-1 signaling pathway also appears to be important for regulating insulin biosynthesis [25]. Mice with $\beta$-cell specific defective expression of Xbp1 exhibited hyperglycemia and glucose intolerance resulting from an increase in $\beta$-cell death and a defect in $\beta$-cell proliferation [8]. The decrease in sXBP1 in VIMP KD cells therefore reduces the ER's capacity to fold new proteins as well as target damaged proteins for retro-translocation out of the ER for degradation. In this study, since IRE1 $\alpha$ is reduced, IRE1-dependent mRNA decay (RIDD) may be disrupted. In VIMP KD cells, decreased PERK causes a decrease in both ATF4 and phosphorylation of eIF2 $\alpha$. The reduction in eIF2 $\alpha$ phosphorylation thereby increases de novo peptide loading onto the ER. ATF 4 can regulate cell fate during ER stress by transcriptional regulation of the expression of its targets involved in amino acid metabolism, oxidoreductases, several ubiquitin ligases and GADD34 phosphatase [23]. Therefore, the decrease in ATF4 and eIF2a in VIMP KD cells may produce deleterious effects. Moreover, UPR can directly regulate cell cycle. IRE1 $\alpha$ can control cyclin A1 expression and promote cell proliferation through XBP-1 [35]. The IRE1 $\alpha$-XBP1 pathway also regulates metabolic stress-induced compensatory proliferation of $\beta$-cells by regulating 
cyclin D1 [36]. In MIN6 cells, VIMP KD may induce the decrease in cyclin D1 through sXBP-1 since both of them are decreased in VIMP KD cells. However, whether UPR inaction induced by VIMP KD can directly or indirectly affect $\beta$-cell death and proliferation need to be further studied. Moreover, VIMP KD increased the insulin production and secretion. Since insulin is necessary for $\beta$-cell survival and proliferation [37], it may be the feedback of the decrease in cell survival and proliferation.

\section{Conclusion}

In summary, our study suggests that VIMP KD induces an increase in $\beta$-cell death and insulin secretion, and a decrease in $\beta$-cell proliferation. VIMP suppression also causes $\mathrm{p} 27$ and Rb-dependent G1 arrest and modulates UPR activation. Therefore, VIMP is a novel selenoprotein regulating $\beta$-cell survival, proliferation and insulin secretion in MIN6 cells.

\section{Acknowledgements}

D.R. designed research; M.L., and J.S. performed research; D.R. analyzed data; and D.R. wrote the paper. The authors thank Dr. K. Polonsky for insightful suggestions, advice and discussions. This study was supported by P30-DK020595 (The University of Chicago and Diabetes Research and Training Center).

\section{Disclosure Statement}

The authors have no conflicts of interest.

\section{References}

1 Butler PC, Meier JJ, Butler AE, Bhushan A: The replication of beta cells in normal physiology, in disease and for therapy. Nature clinical practice Endocrinology \& metabolism 2007;3:758-768.

2 Choi D, Woo M: Executioners of apoptosis in pancreatic \{beta\}-cells: not just for cell death. American journal of physiology Endocrinology and metabolism 2010;298:E735-741.

-3 Tomita T: Apoptosis in pancreatic beta-islet cells in Type 2 diabetes. Bosnian journal of basic medical sciences 2016;16:162-179.

4 Song I, Muller C, Louw J, Bouwens L: Regulating the beta cell mass as a strategy for type-2 diabetes treatment. Current drug targets 2015;16:516-524.

$>5$ Leem J, Koh EH: Interaction between mitochondria and the endoplasmic reticulum: implications for the pathogenesis of type 2 diabetes mellitus. Experimental diabetes research 2012;2012:242984.

-6 Marre ML, James EA, Piganelli JD: beta cell ER stress and the implications for immunogenicity in type 1 diabetes. Frontiers in cell and developmental biology 2015;3:67.

$\rightarrow 7$ Chan JY, Luzuriaga J, Bensellam M, Biden TJ, Laybutt DR: Failure of the adaptive unfolded protein response in islets of obese mice is linked with abnormalities in beta-cell gene expression and progression to diabetes. Diabetes 2013;62:1557-1568.

$>8$ Lee AH, Heidtman K, Hotamisligil GS, Glimcher LH: Dual and opposing roles of the unfolded protein response regulated by IRE1alpha and XBP1 in proinsulin processing and insulin secretion. Proc Natl Acad Sci U S A 2011;108:8885-8890.

-9 Zhang W, Feng D, Li Y, Iida K, McGrath B, Cavener DR: PERK EIF2AK3 control of pancreatic beta cell differentiation and proliferation is required for postnatal glucose homeostasis. Cell Metab 2006;4:491-497.

10 Cavener DR, Gupta S, McGrath BC: PERK in beta cell biology and insulin biogenesis. Trends in endocrinology and metabolism: TEM 2010;21:714-721. 


\section{Cellular Physiology Cell Physiol Biochem 2018;51:2185-2197

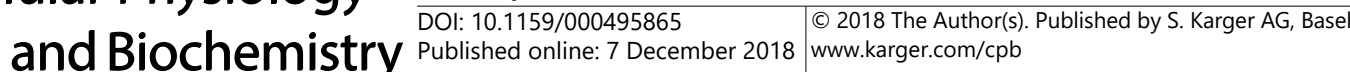

Men et al.: VIMP Deficiency Causes $\beta$-Cell Death

11 Lee JH, Park KJ, Jang JK, Jeon YH, Ko KY, Kwon JH, Lee SR, Kim IY: Selenoprotein S-dependent Selenoprotein K Binding to p97(VCP) Protein Is Essential for Endoplasmic Reticulum-associated Degradation. J Biol Chem 2015;290:29941-29952.

12 Kim KH, Gao Y, Walder K, Collier GR, Skelton J, Kissebah AH: SEPS1 protects RAW264.7 cells from pharmacological ER stress agent-induced apoptosis. Biochem Biophys Res Commun 2007;354:127-132.

13 Curran JE, Jowett JB, Elliott KS, Gao Y, Gluschenko K, Wang J, Abel Azim DM, Cai G, Mahaney MC, Comuzzie AG, Dyer TD, Walder KR, Zimmet P, MacCluer JW, Collier GR, Kissebah AH, Blangero J: Genetic variation in selenoprotein S influences inflammatory response. Nat Genet 2005;37:1234-1241.

14 Du S, Liu H, Huang K: Influence of SelS gene silence on beta-Mercaptoethanol-mediated endoplasmic reticulum stress and cell apoptosis in HepG2 cells. Biochim Biophys Acta 2010;1800:511-517.

15 Olsson M, Olsson B, Jacobson P, Thelle DS, Bjorkegren J, Walley A, Froguel P, Carlsson LM, Sjoholm K: Expression of the selenoprotein S (SELS) gene in subcutaneous adipose tissue and SELS genotype are associated with metabolic risk factors. Metabolism 2011;60:114-120.

16 Gao Y, Feng HC, Walder K, Bolton K, Sunderland T, Bishara N, Quick M, Kantham L, Collier GR: Regulation of the selenoprotein SelS by glucose deprivation and endoplasmic reticulum stress - SelS is a novel glucoseregulated protein. FEBS Lett 2004;563:185-190.

17 Gao Y, Walder K, Sunderland T, Kantham L, Feng HC, Quick M, Bishara N, de Silva A, Augert G, Tenne-Brown J, Collier GR: Elevation in Tanis expression alters glucose metabolism and insulin sensitivity in H4IIE cells. Diabetes 2003;52:929-934.

18 Ren D, Sun J, Wang C, Ye H, Mao L, Cheng EH, Bell GI, Polonsky KS: Role of BH3-only molecules Bim and Puma in beta-cell death in Pdx1 deficiency. Diabetes 2014;63:2744-2750.

19 Tsuru A, Imai Y, Saito M, Kohno K: Novel mechanism of enhancing IRE1alpha-XBP1 signalling via the PERKATF4 pathway. Scientific reports 2016;6:24217.

20 Boyd V, Cholewa OM, Papas KK: Limitations in the Use of Fluorescein Diacetate/Propidium Iodide (FDA/PI) and Cell Permeable Nucleic Acid Stains for Viability Measurements of Isolated Islets of Langerhans. Curr Trends Biotechnol Pharm 2008;2:66-84.

21 Ren D, Sun J, Mao L, Ye H, Polonsky KS: BH3-only molecule Bim mediates beta-cell death in IRS2 deficiency. Diabetes 2014;63:3378-3387.

22 Harding HP, Zhang Y, Bertolotti A, Zeng H, Ron D: Perk is essential for translational regulation and cell survival during the unfolded protein response. Molecular cell 2000;5:897-904.

23 Harding HP, Zhang Y, Zeng H, Novoa I, Lu PD, Calfon M, Sadri N, Yun C, Popko B, Paules R, Stojdl DF, Bell JC, Hettmann T, Leiden JM, Ron D: An integrated stress response regulates amino acid metabolism and resistance to oxidative stress. Mol Cell 2003;11:619-633.

24 Scheuner D, Song B, McEwen E, Liu C, Laybutt R, Gillespie P, Saunders T, Bonner-Weir S, Kaufman RJ: Translational control is required for the unfolded protein response and in vivo glucose homeostasis. Molecular cell 2001;7:1165-1176.

25 Kadowaki H, Nishitoh H: Signaling pathways from the endoplasmic reticulum and their roles in disease. Genes 2013;4:306-333.

-26 Cozar-Castellano I, Fiaschi-Taesch N, Bigatel TA, Takane KK, Garcia-Ocana A, Vasavada R, Stewart AF: Molecular control of cell cycle progression in the pancreatic beta-cell. Endocrine reviews 2006;27:356-370.

-27 Heit JJ, Karnik SK, Kim SK: Intrinsic regulators of pancreatic beta-cell proliferation. Annual review of cell and developmental biology 2006;22:311-338.

28 Lee YC, Nielsen JH: Regulation of beta cell replication. Molecular and cellular endocrinology 2009;297:1827.

29 Lacy ER, Filippov I, Lewis WS, Otieno S, Xiao L, Weiss S, Hengst L, Kriwacki RW: p27 binds cyclin-CDK complexes through a sequential mechanism involving binding-induced protein folding. Nature structural \& molecular biology 2004;11:358-364.

-30 Munoz-Alonso MJ, Acosta JC, Richard C, Delgado MD, Sedivy J, Leon J: p21Cip1 and p27Kip1 induce distinct cell cycle effects and differentiation programs in myeloid leukemia cells. J Biol Chem 2005;280:1812018129.

31 Diehl JA, Cheng M, Roussel MF, Sherr CJ: Glycogen synthase kinase-3beta regulates cyclin D1 proteolysis and subcellular localization. Genes Dev 1998;12:3499-3511. 


\section{Cellular Physiology Cell Physiol Biochem 2018;51:2185-2197

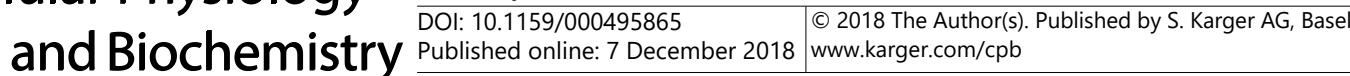

-32 Gesbert F, Sellers WR, Signoretti S, Loda M, Griffin JD: BCR/ABL regulates expression of the cyclindependent kinase inhibitor p27Kip1 through the phosphatidylinositol 3-Kinase/AKT pathway. J Biol Chem 2000;275:39223-39230.

-33 Shin I, Yakes FM, Rojo F, Shin NY, Bakin AV, Baselga J, Arteaga CL: PKB/Akt mediates cell-cycle progression by phosphorylation of p27(Kip1) at threonine 157 and modulation of its cellular localization. Nat Med 2002;8:1145-1152.

-34 Delepine M, Nicolino M, Barrett T, Golamaully M, Lathrop GM, Julier C: EIF2AK3, encoding translation initiation factor 2-alpha kinase 3, is mutated in patients with Wolcott-Rallison syndrome. Nat Genet 2000;25:406-409.

-35 Thorpe JA, Schwarze SR: IRE1alpha controls cyclin A1 expression and promotes cell proliferation through XBP-1. Cell Stress Chaperones 2010;15:497-508.

36 Xu T, Yang L, Yan C, Wang X, Huang P, Zhao F, Zhao L, Zhang M, Jia W, Wang X, Liu Y: The IRE1alpha-XBP1 pathway regulates metabolic stress-induced compensatory proliferation of pancreatic beta-cells. Cell Res 2014;24:1137-1140.

-37 Beith JL, Alejandro EU, Johnson JD: Insulin stimulates primary beta-cell proliferation via Raf-1 kinase. Endocrinology 2008;149:2251-2260. 